\title{
Téoros
}

Revue de recherche en tourisme

\section{La perception des forces et des faiblesses de Montréal comme ville à congrès \\ Selon l'étude Métropoll I}

\section{Pierre Bellerose}

Volume 4, numéro 3, novembre 1985

L'essor de l'industrie des congrès

URI : https://id.erudit.org/iderudit/1080793ar

DOI : https://doi.org/10.7202/1080793ar

Aller au sommaire du numéro

Éditeur(s)

Université du Québec à Montréal

ISSN

0712-8657 (imprimé)

1923-2705 (numérique)

Découvrir la revue

Citer cet article

Bellerose, P. (1985). La perception des forces et des faiblesses de Montréal comme ville à congrès : selon l'étude Métropoll I. Téoros, 4(3), 30-31.

https://doi.org/10.7202/1080793ar d'utilisation que vous pouvez consulter en ligne. 


\title{
La perception des forces et des faiblesses de Montréal comme ville à congrès
}

\author{
par Pierre Bellerose*
}

Durant les cinq dernières années, l'industrie touristique a démontré un intérêt croissant face au marché des congrès et des conférences. Une des manifestations de cet intérêt fut la construction de centres de congrès sophistiqués un peu partout dans le monde. En réponsé à cet intérêt grandissant, le gouvernement fédéral mandatait, en 1984, deux firmes de consultants pour réaliser une étude d'envergure METROPOLL I.

Cette étude dégage les forces et les faiblesses de l'image, de la perception et des facteurs d'attitudes des villes considérées, selon des critẻres bien précis. L'étude s'appuie sur un large échantillon (1650 répondants) de preneurs de décisions des organismes jugés pertinents dans 5 régions géographiques de I'Amérique du Nord. Dans cette étude, les facilités (montage audio-visuel, activités récréatives et touristiques, etc.) reliées aux congrès ont été étudiées du point de vue de l'exécutif d'une association ou d'une corporation qui peut influencer la sélection d'un site pour un congrès ou une conférence. Ce sont donc les perceptions relatives des organismes répondants qui classent et cotent les villes retenues dans l'étude.

\section{Situation de Montréal par rapport aux autres villes concurrentes}

Sur les 75 villes retenues pour les fins de cette étude (au Canada, aux Etats-Unis, aux Caraibes), il appert que les véritables concurrents de Montréal sont ceux se retrouvant dans la même aire géographique désignée "région Est", soit Albany. Atlantic City, Baltimore, Boston, Halifax, Hershey, New York, Philadelphie, Pittsburg. Washington. II est à noter que Toronto n'est pas géographiquement jugée concurrente, mais elle sera citée dans cet article à titre de comparaison.

Sur tous les congrès tenus depuis trois ans par les répondants des diverses associations et corporations, Montréal se situe au

\footnotetext{
- Pierre Bellerose est professeur en tourisme au Cegep de Granby. M. Bellerose est diplômé du baccalauríat on gestion et intervention touristiques de l'UDAM.
}

36 e rang (sur 75 villes) et ses plus proches concurrents de la "région Est" se situent aux rangs suivants: New York au ler rang, Washington au 3eme rang, Boston au 8eme rang. Philadelphie au loeme rang, Baltimore au 21eme rang et Toronto au $24 e ̀ m e$ rang (entre autres).

Deux facteurs viennent cependant fausser quelques peu ces resultats. En fait, Montréal possede son Palais des congrés depuis 1983 alors que l'étude couvre les années 1981 à 1983 précisément. En 1976, le congrès américain a voté une loi pénalisant les congrès d'associations américaines se tenant à l'extérieur des Etats-Unis. Cette loi a été abrogée en décembre 1981 mais on peut supposer que les résultats sousestiment, par rapport à 1985, le score que devraient obtenir les villes canadiennes et plus précisément Montréal.

Si on examine les résultats de cette étude en tenant compte de ces réserves, on constate que la concurrence est très vive sur le marché des congrès. En effet, quand l'on demande aux organisateurs de congres. leurs intentions de tenir un congrès dans les trois prochaines années, la situation demeure à peu près la mëme puisque Montréal récolte le 39 ème rang et Toronto demeure à la 24 ème place. On remarque ici un avantage indéniable au niveau des. congres pour la Ville-Reine, et cela est sans doute grandement dû à son rôle de métropole économique du Canada.

Par rapport à son appartenance géographique (région Est), Montréal se situe au 7ème rang (sur une possibilité de douze) pour les visites passées, au 7 ème rang pour les congrès majeurs tenus depuis les trois dernières années et au Gème rang dans les intentions de tenir un congrès dans les prochaines années. New York, pour les mêmes 3 catégories, se situe respectivement au ler, 2ème et 3ème rang, Boston est au 3ème rang tandis que Philadelphie est au 4eme rang pour les trois catégories. Globalement, Montréal se retrouve dans le deuxième tiers de sa région pour ces catégories.

Pour ce qui est des congrès majeurs planifiés (plus de 300 participants) dans les

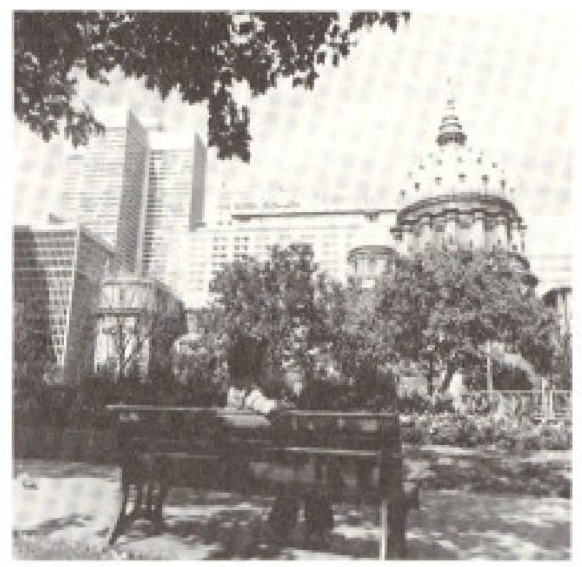

Montréal, ville à congrès de rêputation inter : nationale.

trois années prochaines comparativement aux meetings tenus depuis trois ans, les résultats de l'enquêtte indiquent une baisse de la tenue de congrès à Montréal alors que des villes comme Baltimore, Toronto et Washington devraient connaître une hausse. Cependant, il faut signaler que New York, Boston et Philadelphie vont aussi probablement connaittre une baisse (notons que ces hausses et ces baisses sont des changements estimés selon les réponses soutirées de l'enquête).

Enfin, fait intéressant, cette étude fait ressortir que Montréal se situe au 23 eme rang pour le meilleur travail de publicité et de promotion avec Boston et Washington D.C., derrière Baltimore (22ème rang). Toronto (13ème rang), et New York (6ème rang). On remarque donc que le travail de publicité et de promotion est plus populaire que le choix final des congrès (36ème rangl.

\section{L'image de Montréal}

Si maintenant on s'arrête à la perception de l'image de Montréal, évaluée par les répondants selon 14 critères très importants dans la sélection d ${ }^{r} u n e$ ville pour la tenue d'un congrẻs, Montréal est assez bien percue en rapport avec les autres villes concurrentes de sa région. En examinant le tableau 1 et le tableau 2 , on peut cerner aisément les forces et faiblesses de Montréal au niveau de son image et la compa- 
rer a ses plus proches concurrents, tant pour les congrès que pour les conférences (hôtels). D'après les résultats de ces tableaux, Montréal peut insister par sa publicité et sa promotion sur les critères suivants: hôtels dotés d'installations de con= grés, nombre adéquat de chambres disponibles, bonne cuisine, centre de congrès adéquat, salles et équipements de congrès de qualité, confort des congressistes.

Cependant, Montréal n'est pas bien percue principalement à cause de problèmes liés aux critères suivants: administration compétente, nourriture et logement a prix raisonnable, accessibilité, installation à prix raisonnable, accẻs coûteux, services aériens, pratique personnelle.

Ces derniers critères réfèrent à deux problèmes majeurs, un lié à la position géographique de Montréal et l'autre aux coûts des différents services offerts jugés excessifs. Si une modification de la perception du premier élément laspects géographiques et climatiques/ est difficilement envisageable dans le court ou moven terme. l'autre aspect peut être corrigé en comprenant mieux les mécanismes de marché.

Mais, si l'on compare Montréal avec les 75 villes nord-américaines en isolant le rổle de l'image d'une ville sur le choix du site, on constate que Montréal arrive loeme avec Toronto pour le choix d'un site de conférence et 2lème pour le site d'un congrès (tandis que Toronto arrive $14 \mathrm{~m} \mathrm{me}$ ). En fait, parmi les 12 villes concurrentes qui forment la "région de l'Est", Montréal est bien cotée comme site de conférences l2ème derrière Washington) et comme site de congrès (4eme derrière Washington, Boston et New York avec Philadelphie au meme rang).

En définitive, Montréal est une ville tpès compétitive par rapport à sa région géographique mais comparée aux villes de population comparable, Montréal a une image assez faible surtout comme ville de congrès. Un effort spécial devrait être mis sur le probleme des coûts relatifs aux congrès à Montréal pour permettre d'améliorer sa situation concurrentielle.
Tableau 1

La perception de l'image de Montréal pour l'organisation de séminaires/conférences (hôtels)

\begin{tabular}{lll}
\hline Position de Montreal & Attributs de Montrbal & Villes qui dépassent \\
relative a 20 villes de & les plus compétitifs & Montréal \\
population comparable & dans rEst (total 12) & dans l'Est \\
\hline
\end{tabular}

(Attributs de l'image)

Indice* Rang Indice* Rang

Forces:

1- Salles et équipements de congrès de qualite

2- Popularité (de la ville)

3- Nombre adequat de chambres

$\begin{array}{lllll}143 & 7 & 143 & 3 & \begin{array}{l}\text { Washington } \\ \text { New York }\end{array} \\ 129 & 7 & 175 & 3 & \begin{array}{l}\text { Washington } \\ \text { Philadelphie }\end{array} \\ 121 & 5 & 148 & 5 & \begin{array}{l}\text { Washington } \\ \text { New York } \\ \text { Boston } \\ \text { Philadelphie }\end{array}\end{array}$

4- Près de restaurants de qualite

$\begin{array}{ll}85 & 12 \\ 77 & 14 \\ 62 & 15 \\ 36 & 17 \\ 31 & 18 \\ 72 & 13\end{array}$

5- Accessibilité

6- Equipement audio-visuel

7- Aide à l'organisation

8- Facilités pour la réservation

9- Installations à prix

10- Administration raisonnable

$\left.\begin{array}{ll}110 & 5\end{array}\right] \begin{aligned} & \text { Washington } \\ & \text { Philadelphie } \\ & \text { New York } \\ & \text { Atlantic City }\end{aligned}$

12
14
15
17
18
13

* Movenne $=100$; lorsque sous 90, cela indique un fort problème d'image. Source: Métropoll I (Vol. II).

Tableau 2

La perception de l'image de Montréal pour I'organisation de congrès

\begin{tabular}{lll}
\hline Position de Montréal & Attributs de Montréal & Villes qui dépassent \\
relative à 20 villes de & les plus compótitits & Montréal \\
population comparable & dans l'Est & dans l'Est \\
\hline
\end{tabular}

(Attributs de l'image|
Forces:
1- Salles et equiements
de congrès de qualite
2- Installations modernes
3- Popularite lde la ville)

Faiblesses:

4- Aide à l'organisation

5- Installations à prix raisonnable

6- Nombre adéquat de chambres disponibles

7- Près de restaurants de qualité (hootels)

8- Equipement audio-visuel

9- Facilités pour la réservation

Source: Métropoll I (Vol. II).
* Mayenne $=100$; lorsque sous 90 , cela indique un fort probleme d'image. 